\title{
Síntesis y caracterización de adsorbentes cerámicos basados en polititanatos de potasio y vidrio $\mathrm{SiO}_{2}-\mathrm{B}_{2} \mathrm{O}_{3}-\mathrm{R}_{2} \mathrm{O}-\mathrm{Al}_{2} \mathrm{O}_{3} \mathrm{O}$
}

\author{
MIGUEL ÁNGEL AGUILAR-GONZÁLEZ a,b ALEXANDER GOROKHOVSKY a \\ ALFREDO AGUILAR-ELGUEZABAL b, JOSÉ IVÁN ESCALANTE-GARCÍA a \\ ${ }^{a}$ Centro de Investigación y de Estudios Avanzados, Unidad Saltillo, Coahuila, México \\ ${ }^{\mathrm{b}}$ Centro de Investigación en Materiales Avanzados, Chihuahua México
}

\begin{abstract}
Se fabricaron materiales cerámicos porosos con una composición de 42,5-48,5\% polititanato de potasio (sintetizado en sales fundidas), 1,5-7,5\% de vidrio $\mathrm{SiO}_{2}-\mathrm{B}_{2} \mathrm{O}_{3}-\mathrm{R}_{2} \mathrm{O}-\mathrm{Al}_{2} \mathrm{O}_{3}$ y $50 \%$ de almidón de patata. Las muestras se obtuvieron por extrusión de las pastas, secado y sinterizado a $1100^{\circ} \mathrm{C}$. La caracterización estructural de los materiales se realizó por microscopía óptica y electrónica, difracción de rayos $X$, métodos de porosimetría por intrusión de mercurio y de adsorción/ desorción de nitrógeno sobre la superficie del sólido (BET). La relación óptima del contenido de polititanato de potasio: vidrio fue de 9:1, en base a los mejores resultados de resistencia mecánica. El vidrio funciona como ligante cerámico y participa en la modificación de la estructura del titanato de potasio fibroso obtenido durante la sinterización; la presencia de vidrio transforma la estructura del tetratitanato de potasio, obtenido sin vidrio, en una estructura similar al hexatitanato de potasio. Se encontró que el material sinterizado presenta una superficie específica considerable $\left(1,24 \mathrm{~m}^{2} / \mathrm{g}\right)$, poros de transporte con diámetro promedio de $35 \mu \mathrm{m}$ y meso-poros con diámetro promedio de $2,3 \mathrm{~nm}$. Además, el material producido tiene buenas propiedades de adsorción de metales pesados como el $\mathrm{Pb}$ y puede recomendarse como un adsorbente comercial.
\end{abstract}

Palabras clave: Material adsorbente, titanato de potasio, sinterización, cerámica porosa

Synthesis and characterization of ceramic adsorbents based on potassium polytitanates and $\mathrm{SiO}_{2}-\mathrm{B}_{2} \mathrm{O}_{3}-\mathrm{R}_{2} \mathrm{O}-\mathrm{Al}_{2} \mathrm{O}_{3}$ glass

Porous ceramic materials based on 42.5-48.5 potassium polytitanate (obtained by molten salt synthesis), $1.5-7.5 \mathrm{SiO}_{2}-\mathrm{B}_{2} \mathrm{O}_{3}-$ $\mathrm{R}_{2} \mathrm{O}-\mathrm{Al}_{2} \mathrm{O}_{3}$ glass and 50 potato starch, were produced by extrusion of the pastes, which were further dried and sintered at $1100^{\circ}{ }^{\circ} \mathrm{C}$. The structural characterization of the ceramic materials was carried out by optical and electron microscopy, X-ray diffraction and methods of nitrogen adsorption and mercury intrusion porosimetry. The optimal weight ratio of the potassium polytitanate:glass was 9:1, which was selected based on the highest compressive mechanical of the materials produced. During the sintering process, the presence of the glass promotes the binding of fibrous potassium titanate crystals and favors modification of their structure from potassium tetratitanate (obtained without glass admixtures) to a structure similar to potassium hexatitanate. It is shown that the produced ceramic materials have a high internal surface area (1.24 $\mathrm{m}^{2} / \mathrm{g}$ ) and, in addition to the transport pores with average diameter of $35 \mu \mathrm{m}$, have meso-pores with an average diameter of $2.3 \mathrm{~nm}$. The developed material showed high capability of adsorption of heavy metals like $\mathrm{Pb}$ and could be recommended as a highly efficient adsorbent.

Keywords: Adsorbents, potassium titanate, sintering, porous ceramics

\section{INTRODUCCIÓN}

Los materiales cerámicos porosos poseen un gran potencial para muchas aplicaciones químicas, térmicas y mecánicas entre muchas otras; además, poseen características que les dan ventajas sobre los materiales metálicos o poliméricos. Los cerámicos porosos tienen su principal aplicación como aislantes térmicos, filtros, membranas, materiales adsorbentes y biocerámicos. Los requerimientos para la matriz cerámica y la estructura porosa varían dependiendo del tipo de aplicación; por ejemplo, para aislantes térmicos la porosidad debe encontrarse cerrada, mientras que para los materiales adsorbentes se recomienda utilizar una porosidad abierta.

La remoción de metales pesados de soluciones acuosas, provenientes de procesos industriales tales como la fabricación de: productos textiles, papel, pinturas, baterías, etc., representa una materia importante en el campo de la salud pública y el medio ambiente. Por ejemplo, el plomo daña el sistema nervioso central humano y desbalancea los organismos jóvenes (1). El carbón activado es el material adsorbente que tiene la más alta efectividad de eliminación de metales pesados de aguas negras; sin embargo, su aplicación industrial está limitada por su alto costo (2); por esta razón, en la última decada se han desarrollado intensamente materiales adsorbentes alternativos (3-5).

Un problema vigente es la obtención de materiales adsorbentes que combinen óptimamente su costo y efectividad, y que además posean propiedades mecánicas adecuadas que permitan su fácil manipulación. Los materiales cerámicos tienen buenas propiedades mecánicas y durabilidad 
química; sin embargo, sus propiedades de adsorción no son adecuadas.

Los titanatos de potasio como el $\mathrm{K}_{2} \mathrm{Ti}_{6} \mathrm{O}_{13}$ y especialmente el $\mathrm{K}_{2} \mathrm{Ti}_{4} \mathrm{O}_{9}$, son materiales que han mostrado altas propiedades de adsorción de iones de metales pesados $(\mathrm{Hg}, \mathrm{Cd}, \mathrm{Ba}, \mathrm{Cr}$, $\mathrm{Ni}, \mathrm{Cu}$ y $\mathrm{Co}$ ) en soluciones acuosas (6-8). Por otro lado, los cristales de hexa-titanato de potasio $\left(\mathrm{K}_{2} \mathrm{Ti}_{6} \mathrm{O}_{13}\right)$ presentan buenas propiedades mecánicas. Sin embargo, en dichos trabajos previos los titanatos de potasio se aplicaron como adsorbentes en forma de polvos finos, las cuales no son prometedores para usarlos como materiales adsorbentes comerciales presentados tradicionalmente como gránulos o cilindros.

La producción de materiales adsorbentes comerciales (granulados) de titanatos de potasio cristalinos presenta problemas por la ausencia de fase vítrea en la estructura obtenida después del proceso de sinterizado. Para el caso de probetas cilíndricas cerámicas de titanatos de potasio fibrosos se obtuvieron buenas propiedades mecánicas solamente mediante la aplicación de altas cargas a compresión (9), lo cual excluyó la posibilidad de producir cerámica porosa.

Existen varios métodos para la producción de materiales cerámicos con porosidad abierta, tales como el método de la esponja polimérica (10), en el cual se infiltra una suspensión cerámica en una esponja, se continua con un tratamiento de secado y otro térmico. Existen también procesos en los que se utilizan materiales orgánicos, para producir réplicas de estructuras macroporosas de espumas que contienen poros interconectados (11-14). También se han utilizado agentes que se descomponen o reaccionan para formar estructuras porosas después de un ciclo de tratamiento térmico. Los mejores resultados se han obtenido con la utilización de almidón como formador de estructuras porosas en cuerpos cerámicos, como resultado de un tratamiento térmico (15). Actualmente se producen materiales cerámicos porosos de alúmina, zirconia, titania e hidroxiapatita con excelentes resultados.

En investigaciones previas (9), se reportó el desarrollo de nuevos tipos de titanatos de potasio llamados "polititanatos de potasio" (PTP), obtenidos mediante la ruta de sales fundidas. Los polititanatos de potasio semicristalinos obtenidos son de bajo costo y de fácil manejo en las operaciones comunes de tecnología cerámica; adicionalmente, no representan riesgos para la salud en su manejo debido a su morfología laminar. El tratamiento térmico de dichos materiales a temperaturas desde 900 hasta $1300^{\circ} \mathrm{C}$ permite la recristalización con la formación de tetratitanato y/o hexatitanato de potasio, en función de las relaciones molares relativas de $\left[\mathrm{TiO}_{2}\right] /\left[\mathrm{K}_{2} \mathrm{O}\right]$ en el PTP usado. Esta combinación de tales compuestos permitiría la producción de materiales cerámicos que se caracterizarían por sus altas y controladas propiedades mecánicas, al estar presente el $\mathrm{K}_{2} \mathrm{Ti}_{6} \mathrm{O}_{13}$ y de adsorción, por la presencia de $\mathrm{K}_{2} \mathrm{Ti}_{4} \mathrm{O}_{9}$. Por lo anterior, puede esperarse que los materiales cerámicos porosos, obtenidos a base de polititanatos de potasio con relaciones molares desde 4 hasta 6 de $\mathrm{TiO}_{2}: \mathrm{K}_{2} \mathrm{O}$, podrían ser buenos adsorbentes comerciales ya que se combinarían la estabilidad mecánica y las propieades de adsorción.

Sin embargo, para que los materiales cerámicos porosos posean altas propiedades mecánicas, es deseable la presencia de una fase vitrea que funcione como ligante y ayude a la sinterización. En el presente estudio se desarrolló una técnica innovadora para elaborar materiales cerámicos porosos con buenas propiedades mecánicas y de adsorción a partir de la mezcla de polititanatos de potasio, almidón de patata y vidrio $\mathrm{SiO}_{2}-\mathrm{B}_{2} \mathrm{O}_{3}-\mathrm{R}_{2} \mathrm{O}-\mathrm{Al}_{2} \mathrm{O}_{3}$, por el método de extrusión convencional con un ciclo de tratamiento térmico posterior. .

\section{PARTE EXPERIMENTAL}

\subsection{Materiales}

Se utilizaron las siguientes materias primas para producir los adsorbentes. El PTP se usó como formador de tetratitanato de potasio, como fase principal de material cerámico sinterizado. La síntesis del PTP se realizó de acuerdo con la referencia (9), mediante el tratamiento térmico de una mezcla de $\mathrm{TiO}_{2}$ (anatasa, $10 \%$ ), $\mathrm{KOH}(8 \%)$ y $\mathrm{KNO}_{3}(88 \%)$ a $500{ }^{\circ} \mathrm{C}$ durante 1 hora. Todos los porcentajes referidos en el documento son en peso, al menos que se especifique otra cosa. El producto obtenido se lavó en agua destilada para eliminar vestigios de sales fundidas, posteriormente se secó a $90^{\circ} \mathrm{C}$ durante $2 \mathrm{~h}$ al aire.

Se seleccionó el vidrio de laboratorio tipo Pyrex® (sistema $\mathrm{SiO}_{2}-\mathrm{B}_{2} \mathrm{O}_{3}-\mathrm{R}_{2} \mathrm{O}-\mathrm{Al}_{2} \mathrm{O}_{3}$ ) como ligante cerámico por su alta resistencia química y térmica; el vidrio se procesó previamente en un molino planetario de bolas. El almidón se obtuvo del proceso de lavado de patatas para elaborar frituras (Sabritas Inc.), éste se usó en la mezcla de materias primas como un componente formador de poros. Los materiales se caracterizaron en dos etapas, primero los de partida y posteriormente los cuerpos extruídos con y sin tratamiento térmico. Finalmente se evaluaron sus propiedades de adsorción de plomo en soluciones acuosas de $\mathrm{Pb}\left(\mathrm{NO}_{3}\right)_{2}$.

\subsection{Producción de material cerámico adsorbente}

Las pastas para la fabricación de gránulos de material cerámico en verde se prepararon con $(50-\mathrm{x}) \%$ de PTP, $\mathrm{x} \%$ vidrio $(x=1,5-7,5)$ y $50 \%$ de almidón, con adiciones de $40 \%$ de agua destilada con respecto al peso de los sólidos. Las cantidades de almidón y agua se seleccionaron de acuerdo con (15) como óptimas para producir materiales cerámicos con alta porosidad abierta. La proporción óptima entre el PTP y el vidrio se seleccionó basándose en datos de experimentos preliminares realizados para definir la cantidad mínima de vidrio necesaria para obtener una resistencia mecánica aceptable. Todos los componentes se mezclaron hasta obtener una pasta homogénea y se fabricaron cilindros cerámicos en verde con un extrusor manual. Las dimensiones de los cuerpos extruídos fueron de $0.5 \mathrm{~cm}$ de diámetro por $2 \mathrm{~cm}$ de largo. La primera etapa del tratamiento térmico de los cuerpos cerámicos preparados en verde se realizó a $65{ }^{\circ} \mathrm{C}$ durante 1 hora, con la finalidad de promover la inflamación de los gránulos de almidón (16) y generar espacios en la matriz cerámica. La segunda etapa del tratamiento térmico se realizó a $1100{ }^{\circ} \mathrm{C}$ (9) en un horno eléctrico durante 30 minutos, con una velocidad de calentamiento y posterior enfriamiento de $3{ }^{\circ} \mathrm{C} / \mathrm{min}$.

\subsection{Caracterización}

Para definir el tamaño promedio de partículas de almidón, polititanato de potasio y vidrio, se utilizó un analizador de imágenes (Image Pro Plus) adaptado a un microscopio óptico (Olympus, Vanox HMT3). Los materiales se prepararon dispersando $10 \mathrm{mg}$ de muestra en $2 \mathrm{ml}$ de etanol grado reactivo analítico en baño ultrasónico (Beckman 500) durante 
30 minutos, se tomó una gota de la muestra y se fijó en un portamuestras de vidrio; la muestra se recubrió con oro electrolítico en una evaporadora de vacío (JEOL JEE400). La medición se realizó por el método de intersección de líneas, se tomaron 20 partículas por campo en un total de 20 campos.

La identificación de las fases cristalinas en las materias primas de partida y los materiales sintetizados y sinterizados se realizó por difractometría de rayos X (DRX, Philips, X`pert) de geometría Bragg-Brentano, utilizando la radiación $\mathrm{K}_{\alpha}$ del $\mathrm{Cu}$ y monocromador secundario (filtro de $\mathrm{Ni}$ ) trabajando a 40 $\mathrm{kV}$ y $30 \mathrm{~mA}$. Los registros se efectuaron de 5 a $80^{\circ}(2 \theta)$, con un paso de $0.05^{\circ}(2 \theta)$ y un tiempo de conteo de $5 \mathrm{~s}$. La estructura de los materiales se investigó con un microscopio electrónico de barrido (MEB, Philips XL30, ESEM ambiental, equipado con accesorios de Espectroscopía por dispersión de energía, EDS), operado a $30 \mathrm{kV}$.

La resistencia a la compresión se midió con una máquina hidráulica (Controls, Sercom 7) usando una celda de $15 \mathrm{kN}$ y una velocidad de carga de $100 \mathrm{~N} / \mathrm{s}$. Se ensayaron 6 cilindros de cada muestra de material adsorbente producido con diferentes adiciones de vidrio, se reportó el promedio y la desviación estándar. La densidad de los materiales adsorbentes se determinó utilizando el principio de Arquímedes, utilizando tolueno de $99.5 \%$ de pureza; las mediciones se hicieron a $25^{\circ} \mathrm{C}$ en una balanza analítica (Ohaus, Explorer).

La superficie especifica se determinó en un equipo (QuantachromeAutosorb1C, Asic-xtcd6) utilizando el principio de adsorción/ desorción de una monocapa de nitrógeno en la superficie del sólido, basándose en los métodos de BrunauerEmmet-Teller (BET) y de análisis de micro-poros (MP) (17). La distribución de tamaño de poros fue investigada mediante porosimetría por intrusión de mercurio (PIM, Pore Master 60 GT).

La cinética de adsorción del $\mathrm{Pb}(\mathrm{II})$ presente en las soluciones acuosas se determinó en un espectrómetro de emisión atómica (ICP, Thermoelemental Thermojarrel Ash, modelo Iris Intrepid II). El espectrómetro se calibró con estándares certificados NIST.

\section{RESULTADOS Y DISCUSIÓN}

\subsection{Identificación de los materiales de partida y materiales} en verde

En la Figura 1 se muestran las imágenes de las materias primas de partida. Los datos obtenidos con el de analizador de imágenes indican que el PTP tiene morfología irregular, con partículas laminares (tamaño promedio de $5 \mu \mathrm{m}$ ) formando agregados de hasta aproximadamente $100 \mu \mathrm{m}$ y con un promedio $32 \mu \mathrm{m}$; el almidón presenta morfología elipsoidal con gránulos de tamaños entre 3-100 $\mu \mathrm{m}$ y promedio de $34 \mu \mathrm{m}$, y el vidrio está formado por partículas de morfología angular y tamaño de 3-200 $\mu \mathrm{m}$ y promedio de $64 \mu \mathrm{m}$.

La caracterización por EDS del PTP indicó una relación molar de $\mathrm{TiO}_{2}: \mathrm{K}_{2} \mathrm{O}=4,1$. La composición del vidrio usado determinada por ICP fue: $73,7 \% \mathrm{SiO}_{2}, 12,2 \% \mathrm{~B}_{2} \mathrm{O}_{3}, 5,3 \% \mathrm{Al}_{2} \mathrm{O}_{3^{\prime}}$ $4,8 \% \mathrm{Na}_{2} \mathrm{O}, 3,8 \% \mathrm{~K}_{2} \mathrm{O}, 0,7 \% \mathrm{CaO}$.

\subsection{Determinación del contenido óptimo del vidrio $\mathrm{SiO}_{2}-$ $\mathrm{B}_{2} \mathrm{O}_{3}-\mathrm{R}_{2} \mathrm{O}-\mathrm{Al}_{2} \mathrm{O}_{3}$}

El contenido de vidrio es una variable muy importante en el
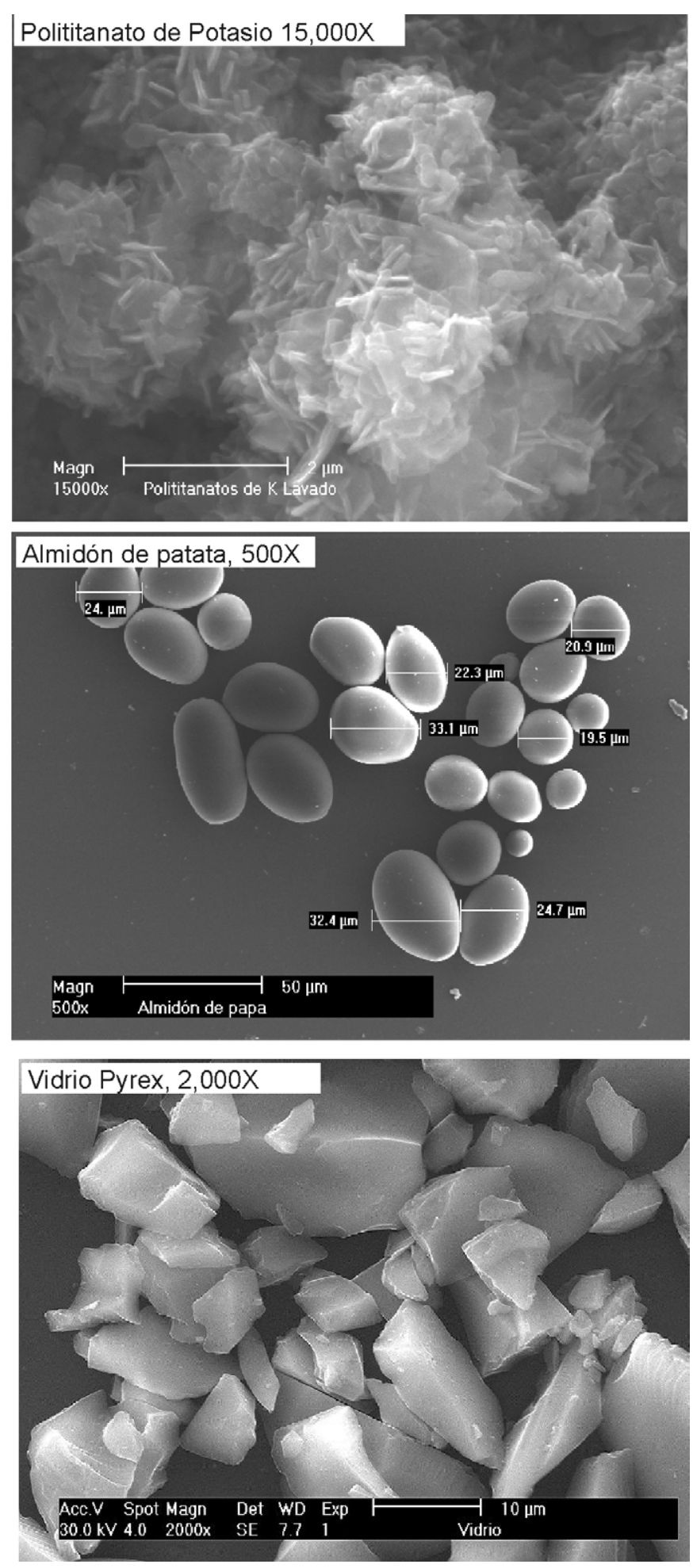

Fig. 1- Micrografías de MEB de los materiales de partida.

material a desarrollar; bajos contenidos no permiten producir materiales porosos con resistencia mecánica adecuada; por otro lado, altos contenidos del mismo reducen las propiedades de adsorción por efecto del bloqueo de la superficie de titanato de potasio. Se fabricaron cuerpos cilíndricos por extrusión usando mezclas de PTP con 1,5, 2,5, 5 y 7,5\% de vidrio y adiciones de $50 \%$ de almidón y $40 \%$ de agua destilada. Las muestras obtenidas se sinterizaron a $1100^{\circ} \mathrm{C}$ con diferentes tiempos de permanencia en el horno. La Figura 2 reporta la influencia del contenido de vidrio y tiempo de tratamiento 


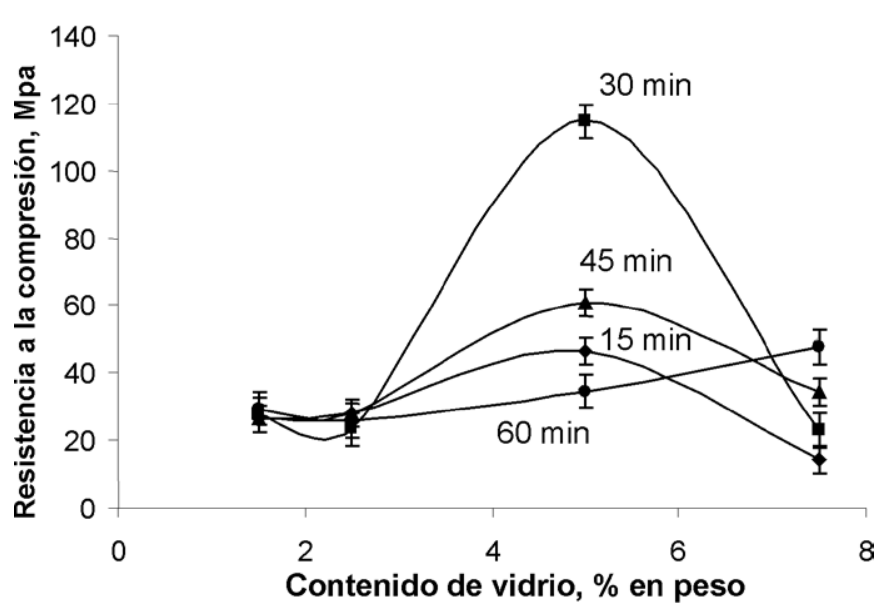

Fig. 2- Influencia del contenido de vidrio y tiempo de sinterización sobre la resistencia mecánica del material.

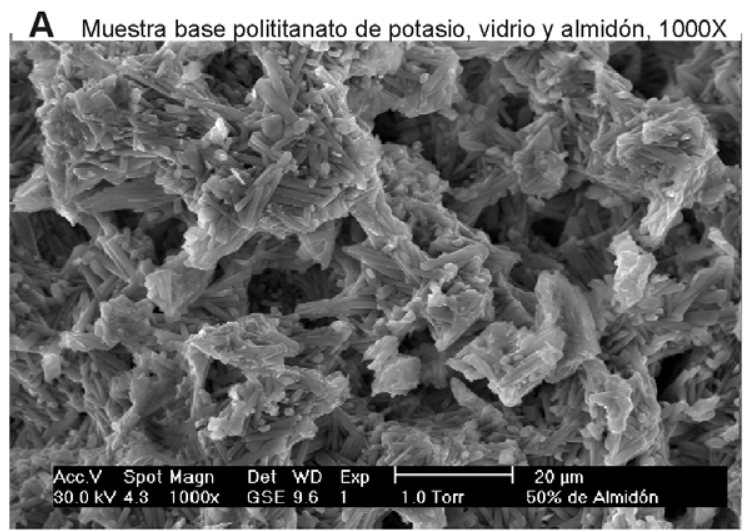

B Muestra base polititanato de potasio, vidrio y almidón, 5000X

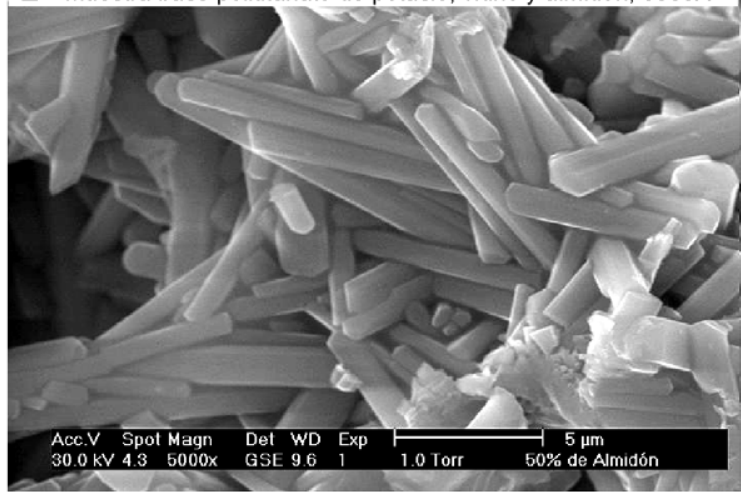

C Muestra base polititanato de potasio y almidón, 5000x

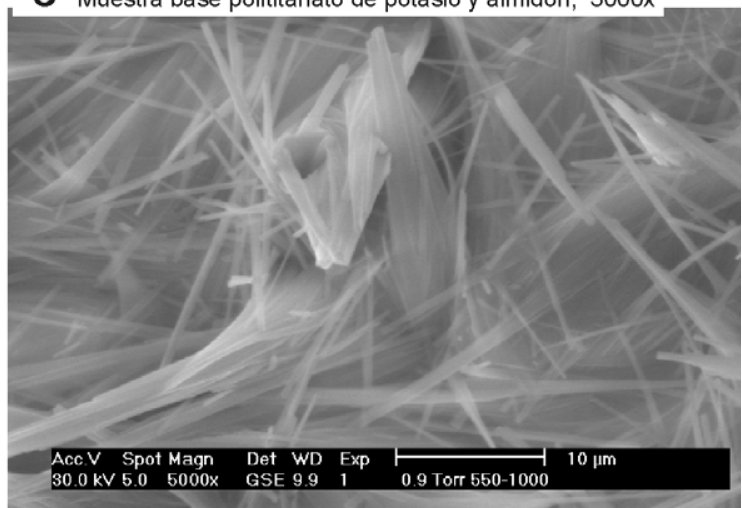

Fig. 3- Microestructura (MEB) de varios materiales sinterizados a $1100^{\circ} \mathrm{C} / 30 \mathrm{~min}$. térmico de sinterizado en el horno sobre la resistencia mecánica. Las muestras con $5 \%$ de vidrio presentaron las mejores propiedades mecánicas; adicionalmente, el tiempo óptimo de sinterización fue de 30 minutos; la resistencia del material preparado en estas condiciones fue de $117 \pm 5 \mathrm{MPa}$. Dicha composición y tiempo de sinterización se seleccionaron para fabricar y caracterizar el material adsorbente.

\subsection{Caracterización}

La Figura 3 presenta la microestructura de los materiales sinterizados, preparados como cilindros extruídos, se compara con un material base PTP procesado con el mismo régimen de tratamiento térmico pero sin adiciones de vidrio. Se puede constatar que los cristales obtenidos con mezcla de PTP y vidrio tienen forma acicular, pero son más gruesos y cortos, en comparación con las fibras delgadas producidas con PTP puro.

El análisis por DRX (Figura 4) indica que el material cerámico producido con la mezcla de PTP y vidrio presenta reflexiones características de hexatitanato de potasio $\left(\mathrm{K}_{2} \mathrm{~T}_{6} \mathrm{iO}_{13}\right)$ pero con un cambio de las intensidades relativas con respecto a la carta de ICDD correspondiente (13-0574). Por otro lado, el material cerámico producido a base de PTP sin vidrio presenta las reflexiones del tetratitanato de potasio $\left(\mathrm{K}_{2} \mathrm{Ti}_{4} \mathrm{O}_{9}\right)$, así también con un cambio en sus intensidades relativas en comparación con la carta de ICDD correspondiente (32-0861).

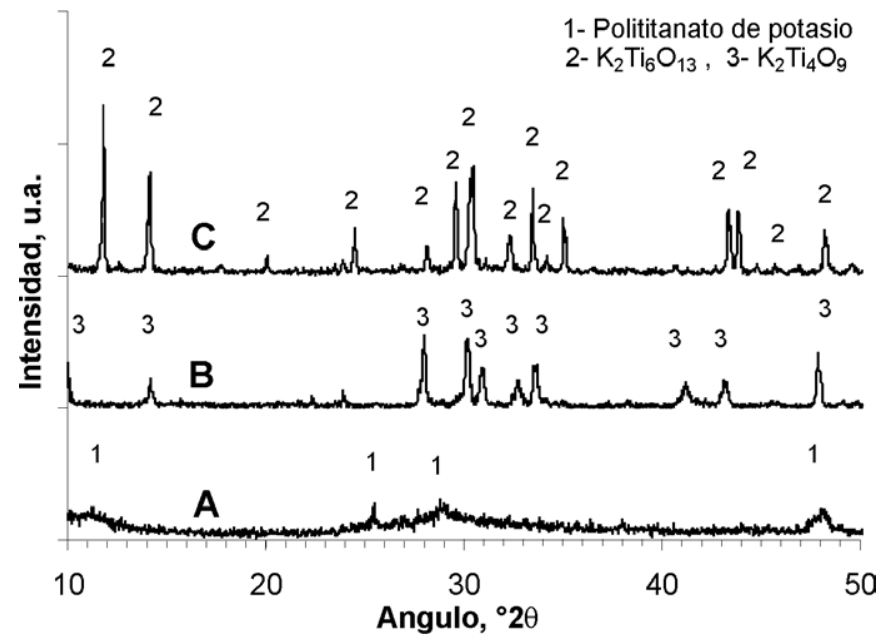

Fig. 4- Difractogramas de rayos $\mathrm{X}$ de $\mathrm{A})$ polititanato de potasio; $\mathrm{B})$ cerámica de polititanato de potasio sinterizada a $1100^{\circ} \mathrm{C} / 30 \mathrm{~min} ; \mathrm{C}$ ) cerámica de polititanato de potasio y vidrio $\mathrm{SiO}_{2}-\mathrm{B}_{2} \mathrm{O}_{3}-\mathrm{R}_{2} \mathrm{O}-\mathrm{Al}_{2} \mathrm{O}_{3}$ sinterizada a $1100^{\circ} \mathrm{C} / 30 \mathrm{~min}$.

De acuerdo con los resultados de EDS, las fases cristalinas del material producido con mezclas de PTP y vidrio, contienen óxidos de titanio $(72-74 \% \mathrm{~mol})$ y potasio $(17-19 \% \mathrm{~mol})$; además contienen $\mathrm{SiO}_{2}(7-9 \% \mathrm{~mol})$. Esto permite proponer que el producto obtenido después de la sinterización presenta una estructura de tetratitanato de potasio, modificada por un dopado de $\mathrm{SiO}_{2}$.

La Figura 5 presenta la isoterma de adsorción de nitrógeno sobre la superficie del material producido (polvo en partículas de 100-150 $\mu \mathrm{m}$ de diámetro). Por hábito de la curva obtenida el adsorbente presentó un carácter típico de los sólidos mesoporosos (17). 


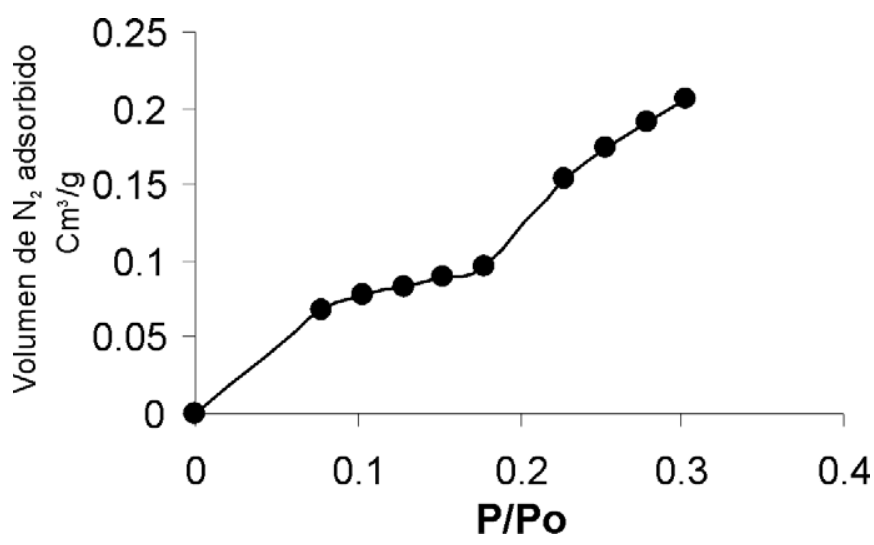

Fig. 5- Isoterma de adsorción de nitrógeno sobre la superficie de material sinterizado.

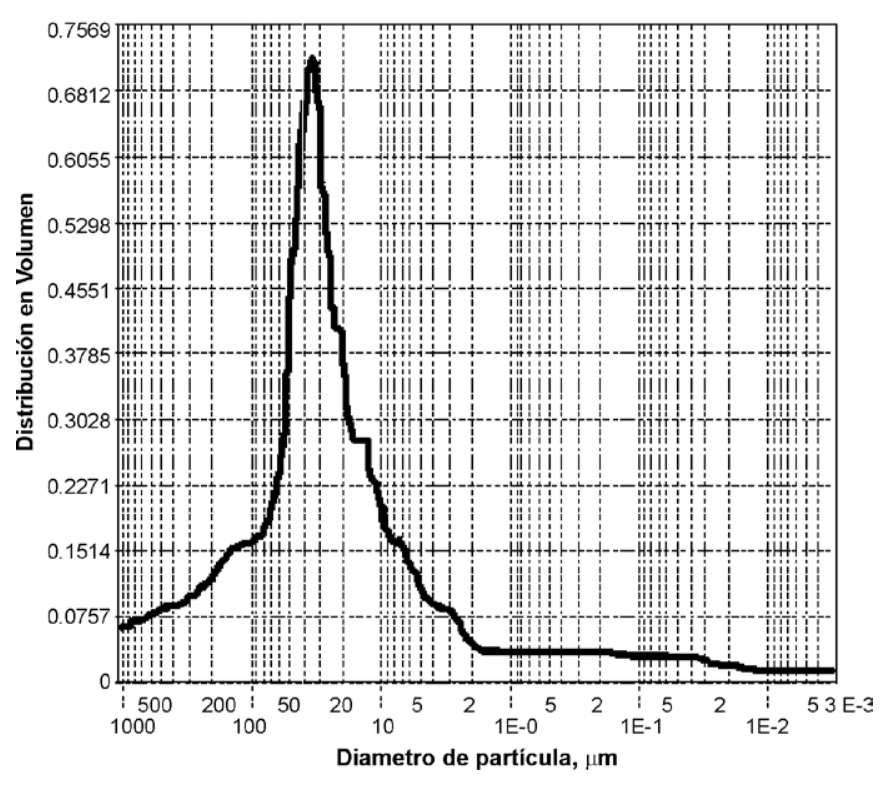

Fig. 6- Distribución de tamaño de poros en el adsorbente sinterizado (datos de porosimetría de vapores de $\mathrm{Hg}$ ).

La superficie específica calculada mediante el método BET fue de $1,236 \mathrm{~m}^{2} / \mathrm{g}$ y se estimó un diámetro de meso-poros de $2,3 \mathrm{~nm}$. La distribución de tamaño de poros (Figura 6) obtenida por la intrusión de mercurio demuestra que, además de los micro- y meso-poros, el material contiene macro-poros de transporte con un diámetro promedio de $35 \mu \mathrm{m}$ que corresponde al tamaño de las partículas del almidón utilizado como materia prima.

La Figura 7 presenta la cinética de adsorción de iones de plomo en la superficie del material después de la inmersión en una solución acuosa de nitrato de plomo cuyo contenido en $\mathrm{Pb}$ corresponde al de los lodos generados por las plantas de tratamiento de aguas negras (92g de $\mathrm{Pb}$ en $\left.1 \mathrm{dm}^{3}\right)$. El material sinterizado demuestra alta efectividad de adsorción de plomo; después de 15 minutos de contacto se redujo la concentración del plomo hasta $1 \mathrm{mg} / \mathrm{dm}^{3}$, el cual es un nivel permitido de acuerdo con la Norma de EPA 55 FR 22520, 1990, USA. El contenido de $\mathrm{K}$ en la solución obtenida aumentó paralelamente con la disminución del contenido de $\mathrm{Pb}$. Esto permite proponer que la adsorción de $\mathrm{Pb}^{2+}$ ocurre mediante un intercambio iónico con los cationes de $\mathrm{K}^{+}$de la estructura de adsorbente.

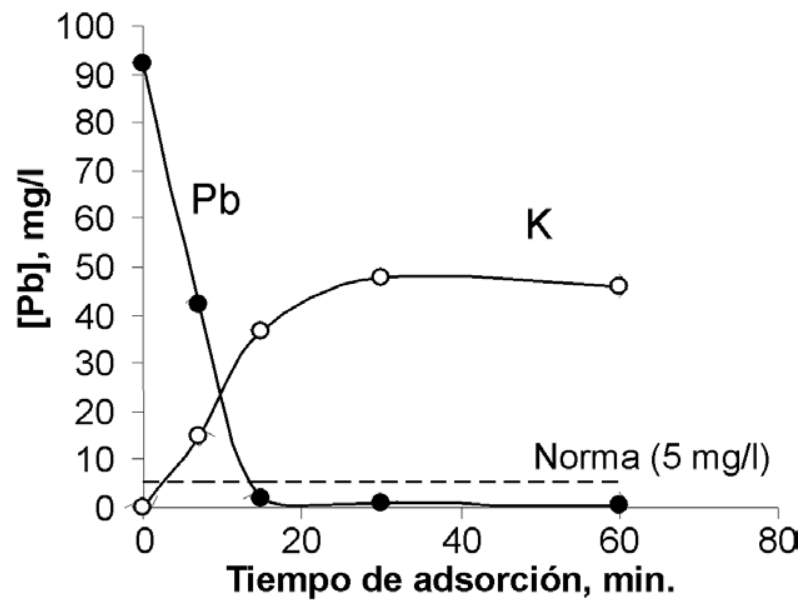

Fig. 7- Influencia del tiempo de contacto con adsorbente (dosis $10 \mathrm{~g}$ / $\mathrm{dm}^{3}$ ) sobre el contenido de $\mathrm{Pb}$ y $\mathrm{K}$ en la solución acuosa de nitrato de potasio.

\section{CONCLUSIONES}

Se han obtenido materiales a partir de pastas extrudidas cuya composición es: $45 \%$ de polititanato de potasio con relación molar de $\mathrm{TiO}_{2} / \mathrm{K}_{2} \mathrm{O}=4,1,5 \%$ vidrio $\mathrm{SiO}_{2}-\mathrm{B}_{2} \mathrm{O}_{3}-\mathrm{R}_{2} \mathrm{O}$ $\mathrm{Al}_{2} \mathrm{O}_{3}$ (Pyrex $($ ) ), 50\% almidón de patata y agua destilada (40\% relativa al peso de los sólidos); los materiales se sinterizaron a $1100^{\circ} \mathrm{C} / 30 \mathrm{~min}$ y sirven para aplicarlos como adsorbentes cerámicos. Éstos presentan en su composición fases de titanato de potasio dopado con $\mathrm{SiO}_{2}$ y una estructura similar a la del $\mathrm{K}_{2} \mathrm{O} \cdot 6 \mathrm{TiO}_{2}$ Dicha estructura se forma durante el tratamiento térmico por una reacción química entre el polititanato de potasio y el vidrio fundido. El resto de la fase vítrea actúa como una matriz ligante en el material cerámico obtenido, favoreciendo una alta resistencia mecánica (aprox. $110 \mathrm{MPa}$ ). El material adsorbente desarrollado tiene una red de poros de transporte (diámetro promedio de $35 \mu \mathrm{m}$ ). La introducción de sílice en la composición de titanato de potasio favorece el incremento del espaciado interlaminar en la estructura cristalina de hasta $2,3 \mathrm{~nm}$. Como resultado, el material demuestra alta velocidad de adsorción de plomo mediante un mecanismo de intercambio iónico con potasio incorporado en la estructura de titanato de potasio dopado.

\section{AGRADECIMIENTOS}

Se reconoce el apoyo financiero del Consejo Nacional de Ciencia y Tecnología (CONACYT, México), mediante el proyecto SEP-CONACYT-2006 No 54883 y la beca otorgada a M.A. Aguilar-González (becario 201784).

\section{BIBLIOGRAFÍA}

1. A.T. Paulino, J.A. Tessari, E.M. Nogami, E. Lenzi, J. Nozaki, Lipid Increase Induced by Lead Accumulation in Tilapia Oreochromis niloticus, Environ. Contam. Toxicol, 75, 1, 42-49 (2005).

2. V.K. Gupta, S. Ali, I.D. Mohan, Equilibrium uptake and sorption dynamics for the removal of a basic dye (basic red) using low-cost adsorbents, J. Colloid Interface Sci, 265, 1, 257-264 (2003).

3. R.P. Han, W.H. Zou, Z.P. Zhang, J. Shi, J.J. Yang, Removal of copper(II) 
and lead(II) from aqueous solution by manganese oxide coated sand - I. Characterization and kinetic study, J. Hazard. Mater, 137, 1, 384-395 (2006)

4. A.T. Paulino, F.A.S. Minasse, M.R. Guilherme, A.V. Reis, E.C. Muniz, J. Nozaki, Novel adsorbent based on silkworm chrysalides for removal of heavy metals from wastewaters, J. Colloid Interface Sci, 301, 2, 479-487 (2006).

5. J.M.Villora, P.Callejas, M.F.Barba, Influencia de procesamiento de materials ceramicos en su comportamiento como adsorbente de iones de aguas industriales, Bol. Soc. Esp. Ceram. V, 43, 1, 75-79 (2004).

6. S.P. Mishra, S.Upadhyaya, Rapid and efficient removal of $\mathrm{Hg}$ (II) from aqueous solution by potassium hexa-titanate. A radiotracer study. J. Radioanal. Nucl. Chem, 189, 2, 247-256 (1995).

7. L.M. Nunes, V.A.Cardoso, C.Airoldi, Layered titanates in alkaline, acidic and intercalated with 1,8-octyldiamine forms as ion-exchangers with divalent cobalt, nickel and copper cations, Mater. Res. Bull, 41, 6, 10891096 (2006).

8. S.Zheng, D.Yin, W.Miao, G.K.Anderson, Cr(IV) photoreduction catalysed by ion-exchangeable layerd compounds", J. Photochem. Photobiol. A Chemistry, 117, 2, 105-109 (1998).

9. A.V.Gorokhovsky, J.I. Escalante-Garcia, T. Sánchez-Monjarás, C.A. GutiérrezChavarría, Synthesis of potassium polytitanate precursors by treatment of $\mathrm{TiO}_{2}$ with molten mixtures of $\mathrm{KNO}_{3}$ and $\mathrm{KOH}$, J.Eur.Ceram.Soc, 24, 13, 3541-3546 (2004).
10. J. Saggio-Woyanski, C.E. Scott, W.P.Minnear, Processing of porous ceramics, Am. Ceram. Soc. Bull, 71, 11, 1674-1682 (1992).

11. J.T. Tian, J.M. Tian, Preparation of porous hydroxyapatite, J. Mater. Sci, 36, 12, 3061-3066 (2001)

12. H.X. Peng, Z. Fan, J. R. G. Evans, J. J. C. Busfield, Microstucture of ceramic foams, J.Eur. Ceram. Soc, 20, 7, 807-813 (2000).

13. J.F. Yang, G.J. Zhang, T. Ohji, Porosity and microstructure control of porous ceramics by partial hot pressing, J. Mater. Res, 16, 7, 1916-1918 (2001).

14. J.M.Villora, P.Callejas, M.F.Barba, C.Baudín, Statistical analysis of the fracture bihaviour of porous ceramic rasching rings, J.Eur. Ceram. Soc, 24, 3, 589-594 (2004).

15. E.Gregorova, W.Pabst, Porosity and pore size control in starch consolidation casting of oxide ceramics-Achievements and problems, J. Eur. Ceram. Soc, 27, 2-3, 669-672 (2007).

16. A. Laurentin, M. Cardenas, J. Ruales, E. Pérez, J. Tovar, Preparation of indigestible pyrodextrins from diferent starch sources, J. Agric. Food Chem, 51, 18, 5510-5515. (2003).

17. D.M.Ruthven, Principles of adsorption and adsorption processes, Wiley Interscience, New York (USA), 1984.

Recibido: 26.06.07 Aceptado: 20.11 .07 